\title{
ON THE MINKOWSKI UNIT OF SLICE LINKS
}

\author{
BY \\ KUNIO MURASUGI
}

1. Introduction. Let $l$ be an oriented polygonal link of multiplicity $\mu$ in 3 -space $R^{3}$ and let $L$ be a diagram of $l$, i.e., an image of a normed regular projection of $l$ onto a plane $R^{2}[9]$. The orientation of $L$ is inherited from that of $l$. To $L$ is associated a symmetric integral matrix $M$, called the symmetric link matrix of $l$ (with respect to $L$ ). It is the symmetrized matrix of the $L$ principal minor of the matrix of $l[8, \S 3]$. If two links are of the same type, then their symmetric link matrices are transformed by a finite sequence of the following two operations and their inverses [8], [10]:

$$
\begin{aligned}
Q_{1}: A \rightarrow R A R^{t}, \text { with } R \text { integral and unimodular, } \\
Q_{2}: A \rightarrow\left(\begin{array}{l|l}
A & 0 \\
\hline 0 & \left.\begin{array}{lll}
0 & 1 \\
1 & 0
\end{array}\right)
\end{array}\right.
\end{aligned}
$$

$R^{t}$ denoting the transposed matrix of $R$.

Now the symmetric link matrix $M$ of a link $l$ may be singular. Then, to $M$ is associated a nonsingular integral matrix $B$ of the highest rank, unless $M$ is a zero matrix (Lemma 2.1). Thus the Minkowski unit $C_{p}(B)\left({ }^{1}\right)$ of $B$ can be defined for any prime integer $p$, including $p=\infty$ (see $\$ 2$ ), and is in fact an invariant of the link type. It will be denoted by $C_{p}(l)$.

The Minkowski unit for a knot, i.e., a link of multiplicity one, was defined by Goeritz only for odd prime integers [4]. Our Minkowski unit for a link is regarded as a generalization of Goeritz's, although the underlying quadratic form is quite different from the one used by him. The particular importance of our quadratic form is that its signature $\left({ }^{2}\right)$ is an invariant of the link type. This enables us to define the Minkowski unit for $p=2$ and $p=\infty$ [5].

Now the concept of a slice link was first introduced by Fox and Milnor for a knot [2] and later Fox generalized it to the case of links [3]. A link $l$ is called a slice link if it is a cross section of a locally flat 2 -sphere in 4-space $R^{4}$. If $l$ bounds a locally flat surface of genus 0 in the half-space $H^{4}\left(^{3}\right)$ of $R^{4}$, then we say that $l$ is a slice link in the weak sense. If $l$ is a slice link, then it is also a slice link in the weak sense, but the converse is not true. Cf. the

\footnotetext{
Received by the editors November 20, 1963.

( $\left.{ }^{1}\right)$ By $C_{p}(B)$ is meant the Minkowski unit $C_{p}(f)$ of the quadratic form $f$ associated to $B$.

( $\left.{ }^{2}\right)$ For definition, see $\$ 2$.

(3) $H^{4}$ is a set of all points in $R^{4}$ whose $x_{4}$-coordinate $\geqq 0$.
} 
remark in §3. In the case of knots, there are no distinctions between them.

Up to the present there are three necessary conditions for a knot to be a slice knot. They are the following:

(1) The Alexander polynomial $\Delta(t)$ must be of the form [2]: $\Delta(t)=f(t) f\left(t^{-1}\right)$.

(2) The Minkowski unit defined by Goeritz must be +1 for any odd prime integer $p[1]$.

(3) The signature of a slice knot must be zero [8].

The main purpose of this paper is to obtain conditions similar to (1) and (2) for slice links.

2. Minkowski unit. In this section some definitions, propositions and lemmas will be given without proofs. For details, for example, see [6] or [7].

Two $n \times n$ symmetric rational matrices $A_{1}$ and $A_{2}$ are said to be $S$ equivalent if one can be obtained from the other by a finite sequence of the operations $Q_{1}$ and $Q_{2}^{ \pm 1}$. If they are transformed by a finite sequence of $Q_{1}^{\prime}$, instead of $Q_{1}$, and $Q_{2}^{ \pm 1}$, then they are said to be $R$-equivalent, where

$$
Q_{1}^{\prime}: A \rightarrow R A R^{t} \text {, with a nonsingular rational matrix } R \text {. }
$$

If two matrices are $S$-equivalent then they are $R$-equivalent.

LeMma 2.1. Any $n \times n$ nonzero symmetric rational matrix $A$ may be transformed by $Q_{1}^{\prime}$ into a matrix of the form

$$
\left(\begin{array}{ll}
B & 0 \\
0 & 0
\end{array}\right)
$$

where $B$ is nonsingular. In particular, if $A$ is an integral matrix, then $A$ may be transformed by $Q_{1}$ into the same form.

$B$ is called a nonsingular matrix associated to $A$. If $A$ is an integral matrix, we may assume that so is $B$. We define the nullity, $n(A)$, of $A$ as $n$ minus the rank of $B$ and $\delta(A)$ as the square free part of $|\operatorname{det} B|$.

(2.1) If $A_{1}$ and $A_{2}$ are R-equivalent, then $n\left(A_{1}\right)=n\left(A_{2}\right)$ and $\delta\left(A_{1}\right)=\delta\left(A_{2}\right)$.

Let $A$ be an $n \times n$ symmetric integral matrix of rank $r$ and $B$ a nonsingular integral matrix associated to $A$.

LEMma 2.2. There is a sequence $B_{1}, B_{2}, \cdots, B_{r}$, called the $\sigma$-series, of principal minors of $B$ such that

(1) $B_{i}$ is of order $i$ and is a principal minor of $B_{i+1}$,

(2) no consecutive matrices $B_{i}$ and $B_{i+1}$ are both singular, for $i=1,2, \cdots, r-1$ $[6, p .9]$.

Let us denote $D_{i}=\operatorname{det} B_{i}$. Then for any prime integer $p$, we define $[6$, p. 31]

$$
c_{p}(B)=\left(-1,-D_{r}\right)_{p} \prod_{i=1}^{r-1}\left(D_{i},-D_{i+1}\right)_{p}
$$


where $(a, b)_{p}$ denotes the Hilbert symbol. If $D_{i+1}=0$, then $\left(D_{i},-D_{i+1}\right)_{p}$ $\times\left(D_{i+1},-D_{i+2}\right)_{p}$ is interpreted to be $\left(D_{i},-h\right)_{p}\left(h,-D_{i+2}\right), h$ being an arbitrary nonzero integer.

(2.2) $c_{p}(B)$ is independent of the choice of $\sigma$-series of $B$.

(2.3) If two nonsingular rational matrices $B_{1}$ and $B_{2}$ can be transformed by $Q_{1}^{\prime}$ from each other, then $c_{p}\left(B_{1}\right)=c_{p}\left(B_{2}\right)$ for any prime integer $p\left({ }^{4}\right)$.

Now following Hasse [5] the Minkowski unit $C_{p}(B)$ for a nonsingular integral matrix will be introduced as follows, $r$ being the rank of $B$.

DeFinition 2.1. For any odd prime integer $p$,

$$
C_{p}(B)=c_{p}(B)(\operatorname{det} B, p)_{p}^{\alpha}
$$

and

$$
C_{2}(B)=c_{2}(B)(-1)^{\beta}
$$

where $\alpha$ denotes the exponent of $p$ occuring in $\operatorname{det} B$, and

$$
\beta=[r / 4]+\{1+[r / 2]\}(d+1) / 2+\left(d^{2}-1\right) m / 8,
$$

[ ] denoting the Guassian symbol $m$ the exponent of 2 occurring in $\operatorname{det} B$ and $d=2^{-m} \operatorname{det} B$.

Finally, for $p=\infty$,

$$
C_{\infty}(B)=\prod C_{p}(B),
$$

product extending over all prime integers.

Let $\sigma$ be the signature of $B$. $\sigma$ is defined by means of the $\sigma$-series of $B$ as follows.

$$
\sigma=\sum_{i=0}^{r-1}\left(\operatorname{sign} D_{i} \cdot \operatorname{sign} D_{i+1}\right),
$$

where sign $D_{i}=D_{i} /\left|D_{i}\right|$ for $D_{i} \neq 0, \operatorname{sign} 0=0$ and $D_{0}=1$. Then we can prove $[5$, p. 223]

$$
C_{\infty}(B)=(-1)^{\gamma},
$$

where $\gamma=[(\sigma-2 v) / 2]+[(\sigma-2 \nu) / 4]$ and $\nu$ denotes the number of odd primes of the form $4 s+3$ occurring with odd powers in the prime factor decomposition of $\operatorname{det} B$.

(2.5) For any odd prime integer $p$ and

$$
B=\left(\begin{array}{ll}
B_{1} & 0 \\
0 & B_{2}
\end{array}\right),
$$

$B_{1}$ and $B_{2}$ being integral and nonsingular,

$\left.{ }^{4}\right) c_{p}(B)$ is not invariant under an operation $Q_{2}^{ \pm 1}$. 


$$
C_{p}(B)=C_{p}\left(B_{1}\right) C_{p}\left(B_{2}\right)\left(\operatorname{det} B_{1}, p\right)_{p}^{\alpha_{2}}\left(\operatorname{det} B_{2}, p\right)_{p}^{\alpha_{1}}\left(\operatorname{det} B_{1}, \operatorname{det} B_{2}\right)_{p},
$$

where $\alpha_{1}$ and $\alpha_{2}$ denote the powers of $p$ occurring in $\operatorname{det} B_{1}$ and $\operatorname{det} B_{2}$, respectively.

Let $A$ be a symmetric integral matrix.

Lemma 2.3. Let $B_{1}$ and $B_{2}$ be nonsingular integral matrices associated to $A$. Then $C_{p}\left(B_{1}\right)=C_{p}\left(B_{2}\right)$ for any prime integer, including $p=\infty$.

Consequently, $C_{p}(A)$ is defined as $C_{p}\left(B_{1}\right)$.

Leмма 2.4. If two symmetric integral matrices $A_{1}$ and $A_{2}$ are $R$-equivalent, then $C_{p}\left(A_{1}\right)=C_{p}\left(A_{2}\right)$ for any prime integer $p$, including $p=\infty$.

Now let $l$ be an oriented link and let $L$ be its diagram. Then as is stated in $\$ 1$, the $S$-equivalent class of symmetric link matrix $M$ of $l$ is a link invariant. Thus from Lemma 2.4, we can define the Minkowski unit $C_{p}(l)$ for $l$ as $C_{p}(M)$ for any prime integer $p$, including $p=\infty$. Moreover, from $(2.1), n(M)$ and $\delta(M)$ are also link invariants, which will be denoted by $n(l)$ and $\delta(l)$ respectively.

3. Slice links. Let $R_{[a, b]},-\infty<a \leqq b<\infty$, be the subspace in 4 -space $R^{4}$ defined as follows:

$$
R_{[a, b]}=\left\{\left(x_{1}, x_{2}, x_{3}, x_{4}\right) \mid a \leqq x_{4} \leqq b\right\} .
$$

In particular $R_{a}^{3}$ means $R_{|a, a|}$.

Now consider a polyhedral and locally flat orientable surface $F$ without singularity in general position in $R^{4}$. Cut it by the family of hyperplanes $R_{t}^{3}$, $-\infty<t<\infty$. We can assume without loss of generality that there are only a finite number of $t$-values that are singular. A singular hyperplane may intersect $F$ in an isolated point, which may be either a maximum or minimum for the height, called an extreme point, or it may intersect $F$ in a graph with just one node, which may be assumed of order four. These nodes are called saddle points.

Let us denote $F_{t}=F \cap R_{t}^{3} F_{t}$ is a link if $R_{t}^{3}$ is not singular.

Let $P$ be a singular point, i.e., an extreme point or a saddle point in $F$. Let $t$ be the height of $P$, i.e., $P \in F_{t}$. Take a sufficiently small $\epsilon>0$ so that $R_{[t-\epsilon, t+\epsilon]}$ $\cap F$ contains no singular points other than $P$. We may assume, hereafter, that none of $F_{t-\epsilon}$ and $F_{t+\epsilon}$ are empty. Thus they are both links.

LEMMA 3.1. If $n\left(F_{t-\epsilon}\right)-n\left(F_{t+\epsilon}\right)= \pm 1$, then

$$
\delta\left(F_{t-\epsilon}\right)=\delta\left(F_{t+\epsilon}\right) \quad \text { and } C_{p}\left(F_{t-\epsilon}\right)=C_{p}\left(F_{t+\epsilon}\right) \text {, }
$$

for any prime integer $p$, including $p=\infty$.

Proof. The multiplicity of $F_{t-\epsilon}$ differs by one from that of $F_{t+\bullet}$. We need only prove the case where the multiplicity of $F_{t-\epsilon}$ is greater than that of $F_{t+c}$. Now the symmetric link matrices $M$ and $M^{\prime}$ of $F_{t-\epsilon}$ and $F_{t+\epsilon}$ can be chosen as follows. (Cf. Lemma 7.1 in [8].) 


$$
M^{\prime}=\left(\begin{array}{c|c}
b & M_{12} \\
\hline M_{21} & M
\end{array}\right), \quad b \text { being an integer. }
$$

Let $B$ and $B^{\prime}$ be nonsingular integral matrices associated to $M$ and $M^{\prime}$. Hence there exist integral unimodular matrices $R$ and $S$ such that

$$
R M R^{t}=\left(\begin{array}{ll}
B & 0 \\
0 & 0
\end{array}\right) \quad \text { and } \quad S M^{\prime} S^{t}=\left(\begin{array}{ll}
B^{\prime} & 0 \\
0 & 0
\end{array}\right)
$$

Since

$$
\left(\begin{array}{ll}
1 & 0 \\
0 & R
\end{array}\right) M^{\prime}\left(\begin{array}{cc}
1 & 0 \\
0 & R^{t}
\end{array}\right)=\left(\begin{array}{c|cc}
b & \multicolumn{2}{|c}{M_{12}} \\
\hline M_{21} & B & 0 \\
0 & 0
\end{array}\right)
$$

and since $B$ is not singular, we can find a nonsingular rational matrix $T$ such that

$d_{i}$ being integers and $s=n(M)$.

$$
T M^{\prime} T^{t}=\left(\begin{array}{c|c|c}
B & 0 & 0 \\
\hline 0 & c & d_{1} \cdots d_{s} \\
\hline & d_{1} & \\
0 & \vdots & 0
\end{array}\right),
$$

Case 1. Suppose that $n\left(F_{t-\ell}\right)=n(M)=s$ and $n\left(F_{t+\varepsilon}\right)=n\left(M^{\prime}\right)=s+1$.

Since the order of $M^{\prime}$ is equal to that of $M^{\prime}$ plus one, the rank of $M^{\prime}$ and that of $M$ are equal. Thus $c, d_{1}, \cdots, d_{s}$ are all zero. Therefore it follows that

$$
\left(\begin{array}{ll}
B & 0 \\
0 & 0
\end{array}\right)=\left(T S^{-1}\right)\left(\begin{array}{ll}
B^{\prime} & 0 \\
0 & 0
\end{array}\right)\left(T S^{-1}\right)^{t} .
$$

Since the order of $B$ and that of $B^{\prime}$ are equal, there is a rational matrix $U$ such that $B=U B^{\prime} U^{t}$ (cf. [7, Lemma 2]). Hence $B$ and $B^{\prime}$ are $R$-equivalent. Thus from Lemma 2.4 it follows that $C_{p}\left(B^{\prime}\right)=C_{p}(B)$, i.e., $C_{p}\left(M^{\prime}\right)=C_{p}(M)$, for any prime integer $p$, including $p=\infty$. Since $\operatorname{det} B^{\prime}=(\operatorname{det} U)^{2} \operatorname{det} B$, it is evident that $\delta\left(M^{\prime}\right)=\delta\left(B^{\prime}\right)=\delta(B)=\delta(M)$.

Case 2. Suppose that $n\left(F_{t-\epsilon}\right)=s$ and $n\left(F_{t+\epsilon}\right)=s-1$.

Since the rank of $M^{\prime}$ is equal to that of $M$ plus two, at least one of $d_{1}, \cdots, d_{s}$, say $d_{1}$, is not zero. Thus there exists a rational matrix $V$ such that

$$
V M^{\prime} V^{t}=\left(\begin{array}{c|cc|c}
B & 0 & 0 & 0 \\
\hline 0 & 0 & d_{1} & 0 \\
0 & d_{1} & 0 & \\
\hline 0 & 0 & 0
\end{array}\right)
$$


Since the order of $B^{\prime}$ is equal to that of $B$ plus two, and since $B$ and $B^{\prime}$ are not singular, we can find a rational matrix $W$ such that

$$
W B^{\prime} W^{t}=\left(\begin{array}{c|cc}
B & 0 & 0 \\
\hline 0 & 0 & d_{1} \\
0 & d_{1} & 0
\end{array}\right) .
$$

Hence $B$ and $B^{\prime}$ are $R$-equivalent. Thus from Lemma 2.4 it follows that $C_{p}\left(B^{\prime}\right)=C_{p}(B)$, i.e., $C_{p}\left(M^{\prime}\right)=C_{p}(M)$, for any prime integer $p$, including $p=\infty$. It is evident that $\delta\left(M^{\prime}\right)=\delta\left(B^{\prime}\right)=\delta(B)=\delta(M)$, q.e.d.

If $P$ is an extreme point, then $n\left(F_{t-\ell}\right)=n\left(F_{t+\ell}\right) \pm 1$. Thus we obtain

Corollary 3.2. If $P \in R_{t}^{3}$ is an extreme point, then

$$
\delta\left(F_{t-\epsilon}\right)=\delta\left(F_{t+\epsilon}\right) \quad \text { and } \quad C_{p}\left(F_{t-\epsilon}\right)=C_{p}\left(F_{t+\epsilon}\right)
$$

for any prime integer $p$, including $p=\infty$.

Leммa 3.3. Let $F$ be a locally flat 2-sphere in $R^{4}$ and let $P$ be a singular point in $F_{t}$. Then

$$
\delta\left(F_{t-\epsilon}\right)=\delta\left(F_{t+\epsilon}\right) \quad \text { and } \quad C_{p}\left(F_{t-\epsilon}\right)=C_{p}\left(F_{t+\epsilon}\right),
$$

for any prime integer $p$, including $p=\infty$.

Proof. From (9.16) in [8], it follows that $\sigma\left(F_{t-\epsilon}\right)=\sigma\left(F_{t+\epsilon}\right)$. Thus Lemma 7.1 (2) in [8] implies that $n\left(F_{t-\epsilon}\right)=n\left(F_{t+\varsigma}\right) \pm 1$. Therefore the Lemma follows from Lemma 3.1.

From this lemma, we obtain the following

TheOREM 3.4. If $l$ is a slice link, then

$$
\delta(l)=1 \quad \text { and } \quad C_{p}(l)=1,
$$

for any prime integer $p$, including $p=\infty$.

Proof. From Lemma 3.3 it follows that $\delta(l)=\delta(\iota)$ and $C_{p}(l)=C_{p}(\iota)$ for any prime integer $p$, including $p=\infty$, where $\iota$ denotes a trivial knot. Since $\delta(\iota)=1$ and $C_{p}(\iota)=1$, the theorem follows immediately.

CoRollary. 3.5. If $k$ is a slice knot, then $\Delta(-1)$ is a square and $C_{p}(k)=1$ for any prime integer $p$, including $p=\infty$.

REMARK. There is a slice link in the weak sense such that $C_{2}=-1$.

Consider the torus link $l$ of the type $(2,2 p), p$ being a prime integer of the form $8 s \pm 3$. Give an orientation to each component so that the symmetric link matrix $M$ of $l$ is given by an $1 \times 1$ matrix (2p). Then

$$
C_{q}=(-1,-2 p)_{q}(2 p, p)_{q}=(-1,-1)_{q}(2 p,-p)_{q}=(-1,-1)_{q}(2,-p)_{q} .
$$

Therefore $C_{2}=-1$ and $C_{p}=-1$. 


\section{REFERENCES}

1. J. J. Andrews, The Minkowski unit of slice knots, Abstract 599-20, Notices Amer. Math. Soc. 10 (1963), 253.

2. R. H. Fox, A quick trip through knot theory, Topology of 3-manifolds and related topics (Proc. The Univ. of Georgia Institute, 1961), pp. 120-167, Prentice-Hall, Englewood Cliffs, N. J., 1962.

3. , Some problems in knot theory, Topology of 3-manifolds and related topics. (Proc. The Univ. of Georgia Institute, 1961), pp. 168-176, Prentice-Hall, Englewood Cliffs, N. J., 1962.

4. L. Goeritz, Knoten und quadratische Formen, Math. Z. 36 (1933), 647-654.

5. H. Hasse, Uber die Aquivalenz quadratischen Formen in Körper der rationalen Zahlen, Crelle J. 152 (1923), 205-224.

6. B. W. Jones, The arithmetic theory of quadratic forms, The Carus Math. Monographs No. 10, The Mathematical Association of America, Buffalo, N. Y., 1950.

7. R. H. Kyle, Branched covering spaces and the quadratic forms of links, Ann. of Math. (2) 59 (1954), 539-548.

8. M. Murasugi, On a certain numerical invariant of link types, Trans. Amer. Math. 117 (1965), 387-422.

9. K. Reidemeister, Knotentheorie, Chelsea, New York, 1948.

10. H. F. Trotter, Homology of group systems with applications to knot theory, Ann. of Math. (2) 76 (1962), 464-498.

\section{Princeton University,} Princeton, New Jersey 